\title{
sciendo
}

DOI 10.2478/sbe-2019-0044

SBE no. 14(3) 2019

\section{A PROPOSAL FOR AN AUTOMATIC STABILIZER IN SOCIAL JUSTICE}

\author{
DINGA EMIL \\ Romanian Academy, Bucharest, Romania \\ TĂNĂSESCU CRISTINA \\ Lucian Blaga University of Sibiu, Romania \\ IONESCU GABRIELA-MARIANA \\ SCOSAAR, Bucharest, Romania
}

\begin{abstract}
:
Generally, social justice has two sides which are intercorrelated and inter-dependend: a) constitutive social justice (for example, the so called commutative social justice); b) regulative social justice (for example, the so called distributive social justice). The paper approaches the regulative social justice, more exactly, an automatic mechanism to get it. To this end, an automatic stabilizer to provide distributive social justice, according to the Rawlsian principle of difference. Such an automatic stabilizer is grounded on the wealth, more precise, on the share of the wealth which is not invested in order to benefit to the more disadvantaged class of the society. Paper does not test empirically the proposal, such a propose remaining for next intervention in this publication.
\end{abstract}

Key words: social justice, automatic stabilizer, difference principle, commutative social justice, distributive social justice

\section{Identification of the design elements of the automatic stabilizer in social justice}

Like any automatic stabilizer (McKay, 2013), the automatic stabilizer in social justice (ASSJ) needs five design elements: a) the activity (economic or social) that generates the variations of interest for the adjustment; b) the base of action of the ASSJ; c) the sensitivity grid of the ASSJ; d) the action rate of the ASSJ; e) the triggering thresholds, respectively of stopping the operation of the ASSJ.

(a) generating activity (GA)

In the field of social justice (remember that, for the design of automatic stabilizers in social justice, we only consider distributive justice) the generating activity regarding the automatic stabilizers is the commutative justice itself. As it is known, the commutative justice refers to the way in which the distribution (or primary distribution) of the social product is made (in 
this case, the economic product measured as GDP). Although the commutative justice cannot be subject to automatic stabilizers but, in our opinion, it is totally accessible only to discretionary decisions, it is the one that, by establishing the primary distribution of the social product can have the effect of changing the base of action of the society from the perspective of the two generic categories of individuals: favoured, that is, located in the upper "pole" of the possession of wealth or income, respectively disadvantaged, that is, located in the lower "pole" of the possession of wealth or income (although a finer structuring of the society is useful in research, for example, a middle area, located between the two poles - the so-called middle class - for the analysis needs in this study, we will only accept the two poles for positioning individuals). Although the primary distribution is, of course, fair, the end result of the distribution of the social product may be, from a broader societal perspective, unfair. This is the reason that causes the activity of generating the need for an automatic stabilizer in the field of social justice (Nussbaum,2007) to be precisely the effect of the commutative justice action.

(b) action base (B)

The base of action of an ASSJ can be any aspect of commutative justice. Thus, if we limit ourselves to the fundamental aspect of this category of social justice (Rawls, 2011), this can only be the current income, associated with the contribution to obtaining the social product (in this case, GDP). We notice immediately that if we wish to consider wealth, this is only the treasured aspect of current income, which means that both the size and rate of change of assets are functionally dependent on the size and rate of change of current income. Of course, wealth is also dependent on the marginal propensity towards saving, but this propensity is, in turn, a function of current income. We can therefore consider that current income is the fundamental element that leads to the emergence, maintenance and variation of economic inequality in society. Inheritance cases or fortuitous cases (such as an unexpected gain in legal pyramid schemes - lotteries - that is, those pyramid schemes that are not Ponzi type), although they can occur, they are not theoretically representative, because they have not predictable repeatability. Thus, if we note with $V_{i}$ the current income in year $i$, with $\beta_{i}$ the marginal propensity towards saving in year $i$, and with $W_{i}$ the wealth in year $i$, then we can write: $W_{i+1}=V_{i} * \beta_{i}$. On a horizon of time $T$, one can write: $W_{T}=\sum_{i=1}^{T}\left(\beta_{i} * V_{i}\right)$. If the marginal propensity towards saving remains constant, the relation becomes: $W_{T}=\beta \cdot \sum_{i=1}^{T}\left(V_{i}\right)$. Although, of course, the phenomenon of accumulation of wealth is much more complicated (investments made from savings should be taken into account, either real investments or portfolio investments, as well as the currency structure of monetary holdings, which leads to asset appreciation or impairment, etc.), essentially the mechanism of wealth differentiation is dependent on the mechanism of income differentiation. Therefore, we will assume that by the action base of an ASSJ we will understand the current income, namely the (just) insured by the commutative justice.

(c) sensitivity grid $(\underline{G})$

By sensitivity grid we mean the distribution, on the "surface" of the action base, of the trigger thresholds, respectively of stopping the ASSJ action. The sensitivity grid therefore represents a linear grid or, as the case may be, a surface grid (or hypersurface - especially in cases where ASSJ chains are designed - depending on the type of ASSJ). It may be "finer", if a more frequent triggering of the stabilizer action is desired, or a "coarser" one if a 
less frequent triggering of the stabilizing action is desired. The grid can be regular (triggering of the stabilizer action is made at equal variations of the action base) or irregular (triggering of the stabilizer action is done at unequal variations of the action base).

Theoretically, the irregular sensitivity grid seems more appropriate, because the variations of the action base have different significance, from an economic and social perspective, depending on the size of the action base (the so-called base effect). The sensitivity grid of an ASSJ is entirely at the latitude of political factor's discretion that designs the ASSJ in question and serves the economic or social policy interest of this political factor. Although certain logical, psychological, institutional or moral considerations (Nozick, 2007) can be taken into account in designing the sensitivity grid, this is overwhelmingly the result of a policy evaluation or even a political evaluation. Figure 1 shows three categories of grid, applied on the base of action.

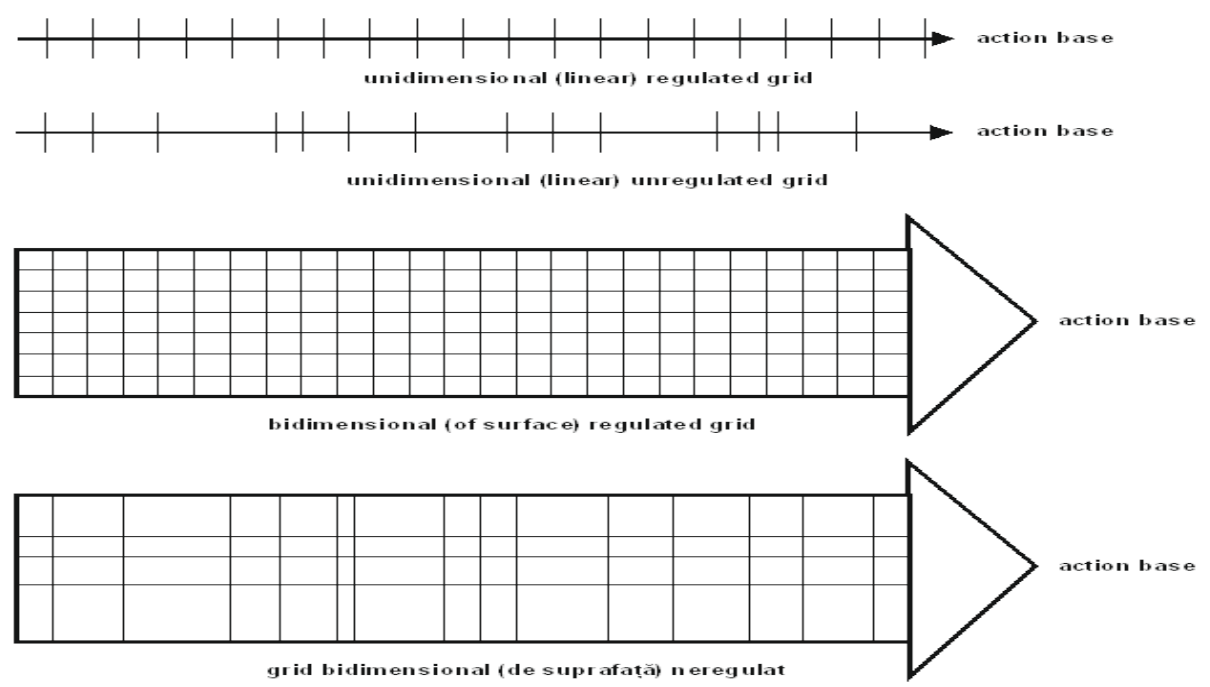

Figure 1. Varieties of the ASSJ sensitivity grid

Source: graphic design of the authors.

(d) action rate $(r)$

The action rate is the intensity with which ASSJ acts on the base of action, according to the "program" incorporated when it was designed. From the instrumental point of view, the exercise of the action rate has the effect of reducing the value of the action base, when it has exceeded, in increasing, the triggering threshold of ASSJ, respectively it has the effect of increasing the action base, when it has exceeded, in decrease, the triggering threshold of the ASSJ (as shown above, the ASSJ must always have a counter-cyclical action in relation to the recorded variation of the base of action. As with the sensitivity grid, the design of the action rate depends on the interest economic or social policy of the political decision maker (more precisely the decision factor that takes the discretionary measure of ASSJ design). From a methodological point of view, the action base represents the quantitative variable of the ASSJ action, the action rate represents the qualitative variable of the ASSJ action, and the grid of sensitivity represents the structural side of the ASSJ action. In the case, for example, of an analysis of the impact of the action of an ASSJ, the 
contribution of the qualitative and quantitative variables can be calculated, between moments 0 and 1 , as follows:

$$
\begin{gathered}
\Delta A^{r}=\Delta r \cdot B_{1} \\
\Delta A^{B}=r_{0} \cdot \Delta B \\
\Delta A=\Delta A^{r}+\Delta A^{B}=r_{1} \cdot B_{1}-r_{0} \cdot B_{0}
\end{gathered}
$$

More generally, that is, if the impact of the simultaneous change of the rate of action and of the base of action is determined separately, the above relations become:

$$
\Delta A=\Delta A^{r}+\Delta A^{B}+\Delta A^{r_{*} B}=r_{0} \cdot \Delta B+\Delta r \cdot B_{0}+\Delta r \cdot \Delta B
$$

From the graphical point of view, the factorial analysis of the quantity of action of an ASSJ is represented in figure 2.

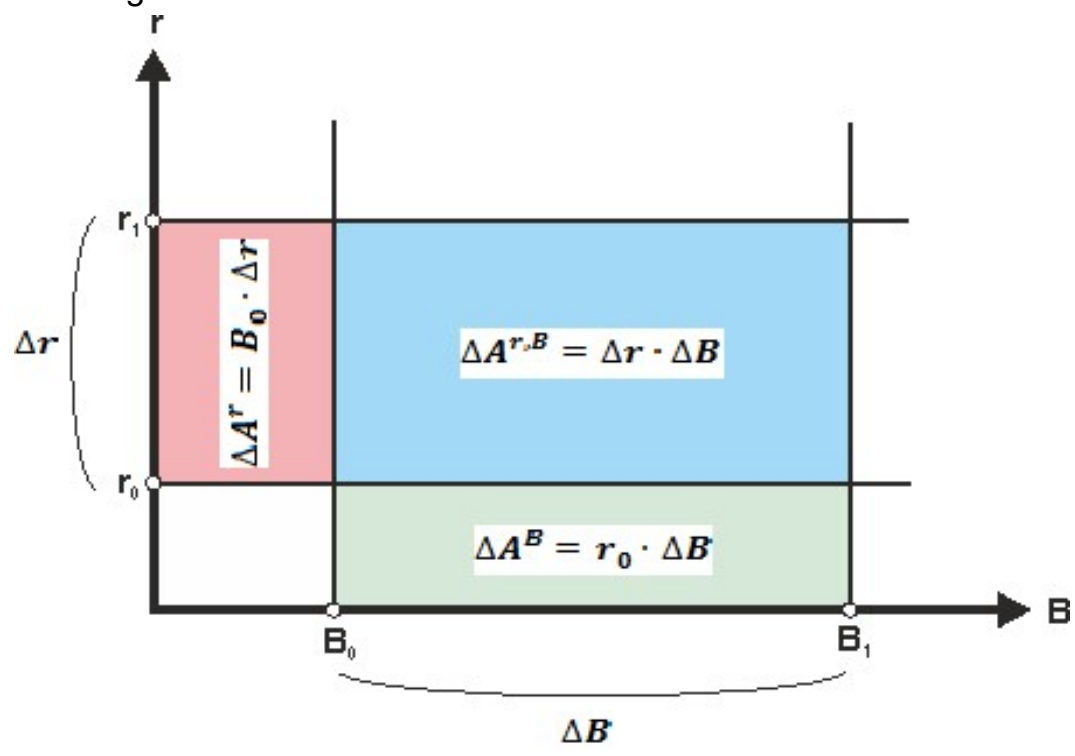

Figure 2. Factor analysis of the variation of the quantity of ASSJ action Source: graphic design of the authors.

(e) thresholds (T)

The threshold problem is, in principle, solved with the design of the sensitivity grid. Thus, the lower limit of a grid "cell" (whether it is one-dimensional or of some size, k) constitutes the threshold for triggering the ASSJ action. As the upper limit of the cell of order " $k$ " is the same as the lower limit of the cell of order "k + 1", it turns out that, in fact, the sensitivity grid of an ASSJ is a planning of the thresholds for triggering the action of the automatic stabilizer in question. On this base, we have nothing specific to add to the thresholds of an ASSJ.

\section{Qualitative evaluation of the design elements of the automatic stabilizer in social justice}

From the point of view of qualitative analysis, only three of the five design elements of an ASSJ are of interest: a) the base of action; b) the sensitivity grid; c) the rate of action.

(a) base of action (B)

The base of action (which is the quantitative, extensive element of an automatic stabilizer) 
(Russek, 2015) can, in principle, be any variable (usually macroeconomic) that is of interest to economic and social policy makers, in the sense of a non-discretionary adjustment interest. At the same time, the design of an automatic stabilizer can be done effectively only if the envisaged action base is compatible with a certain kinematics, or, more precisely, if the activity associated with it can generate an observable variation of that base action. A relatively inert base of action or having a high value of the coefficient of inertia (or rigidity) will not work within the mechanism of an automatic stabilizer. In addition, the base of the action in question must be discretionary adjustable (both methodologically and institutionally) by the designer of the automatic stabilizer. Therefore, the discretionary accessibility of the action base is the sine qua non condition of its automatic accessibility. It is inconceivable that a certain base of action may not be accessible discretely, but be automatically accessible.

(b) sensitivity grig $(\underline{G})$

The whole "philosophy" of an automatic stabilizer is contained in the sensitivity grid. It gives, first, the frequency with which a certain automatic stabilizer will be triggered to adjust the inappropriate variation (from the perspective of the economic or social policy decision maker) of the base of action and, secondly, indicates the scale of use of the automatic stabilizer (the limits between which the variation of the action base is considered automatically adjustable). From a qualitative point of view, the sensitivity grid is indispensable for the operation of an automatic stabilizer. The absence of the grid is equivalent to the de facto abolition of the automatic stabilizer in question, so leaving the decision in the matter of the respective base of action at the discretionary latitude of the decision factor. In fact, from the perspective of designing an automatic stabilizer (and even more so in the case of a field as sensitive as that of social justice), after the purely nominal choice of the base of action, the first design element itself (i.e. the first element in which the intention of adjustment is "captured") is the grid of sensitivity.

(c) action rate $(r)$

The action rate is, as already suggested above, the qualitative element of the mechanism of an automatic stabilizer. The action rate expresses the intensity with which the automatic stabilizer in question performs the adjustment of the action base, at the "command" of the sensitivity grid. The special relevance of the action rate is that it must be designed in such a way that, in the sense that it adjusts the action base (counter-cyclical), as well as by the intensity (or "acceleration") with which it performs this adjustment, it ensures the sustainability of both the action base and the activity that generates the variation of the action base. So, if the base of action is a relatively easy choice for the policy maker (being much closer to a political choice - the political choice is a choice with a minimal degree of grounding, resulting, in most cases, from the shared social ideology or philosophy by the deciding factor), the sensitivity grid and the action rate represent choices of much greater responsibility and methodological difficulty. 


\section{Quantitative evaluation of the design elements of automatic stabilizer in social justice}

In this paragraph we will undertake a brief assessment of the quantitative aspects of the three design elements of an ASSJ that have been examined also from a qualitative perspective.

(a) base action $(\underline{B})$

The variation of the base of action has quantitative limits arising, on the one hand, from the nature of that base of action, and, on the other hand, from the constraints associated with a free and democratic society. For example, if the base of action is the disposable income (remember that the disposable income is the difference between the personal income and the direct tax deducted from the personal income. The disposable income can only be used in two directions: a) final consumption; b) saving), below a certain level, ASSJ can no longer act. In addition, the available income cannot be negative or, if negative, the ASSJ cannot act on it again. This case is obviously a particular case of the one where a minimum value is established below which the disposable income can no longer be subject to ASSJ action. Thus, the quantitative aspects of the base of action refer, in particular, to the economic and social significance of taking into account certain limits (lower and / or higher) in which the base of action may be subject to ASSJ action.

(b) sensitivity grid $(\underline{G})$

Regarding the quantitative aspects of the sensitivity grid of an ASSJ, we consider the following points of interest:

- the analyticity of the sensitivity grid is essential for the functioning of the ASSJ. By analyticity (or, more precisely, by the degree of analyticity) of the grid, we mean the "clearing" of the institutional grid in question, or, what is equivalent, the frequency with which the ASSJ is triggered as the base of the action changes. In this sense, too high a degree of analyticity will lead to too frequent an action of the ASSJ, which can induce instability in the social system, and, symmetrically, a too low degree of analyticity will lead to a rare action of the stabilizer, of nature to strengthen the social system, generating a great rigidity from the perspective of the base of action in question;

- it is a relatively difficult question whether the "grid lines" have to be numbers or intervals, that is, whether the automatic stabilizer programmed through the grid has the sensitivity to be triggered by simply checking a number (point value) regarding the "red line" crossing on the part the action base, or if the variation of the action base should go through a certain range of variation for the automatic stabilizer to be triggered. Such a precaution is necessary, for example, in the event of accidental and reversible overruns of that "red line", in which case, in order to be sure that the variation of the base of action is certain and definitive, the thresholds planned within the grid should be sensitivity of the interval type, not of the punctual one.

(c) rate of action $(r)$

The quantitative aspect of the rate of action concerns, of course, the numerical size by which the rate of action "returns" the base of action from its autonomous variation (in fact, generated by the variation of the associated activity, as shown above). Based on those 
discussed on the principle of sustainability as a sufficient predicate of an ASSJ, we believe that the size of the action rate is a crucial element of ensuring the sustainability of the adjustment of the action base. This size depends not only on the interest of adjusting the policy makers, but also has to take into account some important aspects such as: organizational culture, values and traditions, contagion effects, expectations, etc. As already shown, together with the variation of the action base, the variation of the action rate generates the quantity of action, i.e. the size with which the action base is effectively adjusted. Of course, given that we assumed that $r=r(B)$, it turns out that the intensity of adjustment depends, in a generic way, on the grid chosen for the variation of the base of action.

\section{Mechanism of operation of the automatic stabilizer in social justice}

Regarding the mechanism of operation of an ASSJ, we will describe two aspects: a) the way in which the rate of action (i.e., the variation of the rate of action) is distributed on the sensitivity grid; b) how the variation of the base of action is "reversed" by the action of the rate of action.

(a) although, indeed, the variation of the rate of action is a function of the variation of the base of action, that is to say the "tranches" in which the dimensional value of the base of action is divided, the level of the action rate also depends on the interest of automatic stabilization of the base of action. This means that the intensity with which the action rate acts depends also on a factor outside the action base. We could say that, in fact, the action rate depends on two factors, a methodological one (the degree of analyticity of the action base) and a political one (the desired intensity regarding the impact of the automatic stabilizer in question). From the graphical point of view, and considering only the variation of the action base as a causal factor of the action rate programming, this aspect of the ASSJ mechanism is represented synoptically in figure 3.

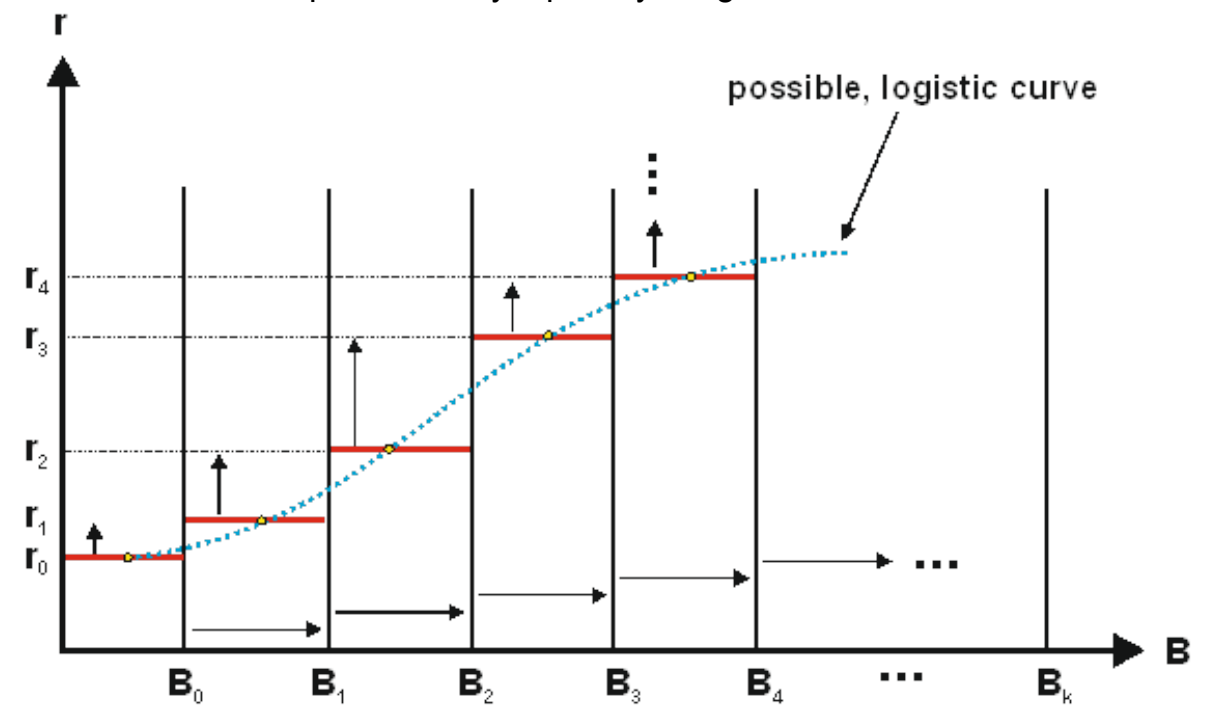

Figure 3. Programming of the action rate according to the variation of the ASSJ action base

Source: graphic design of the authors. 
(b) the "return" of the variation of the base of action as a result of the variation of the action rate represents the end (and, regarding the economic or social policy decision maker, the purpose) of designing and implementing the ASSJ. This means that part or even the entire autonomous variation of the base of action (depending on the intensity of the ASSJ action, the given intensity, as we have seen, the level of the action rate) is cancelled by the ASSJ action. As it is a policy instrument, the ASSJ must be carefully designed, first of all at the methodological level, because its action, being automatic (so independent of the policy-maker), can no longer be corrected, if the design is wrong, by a discretionary decision. Figure 4 shows the impact of the ASSJ action on the variation of the action base.

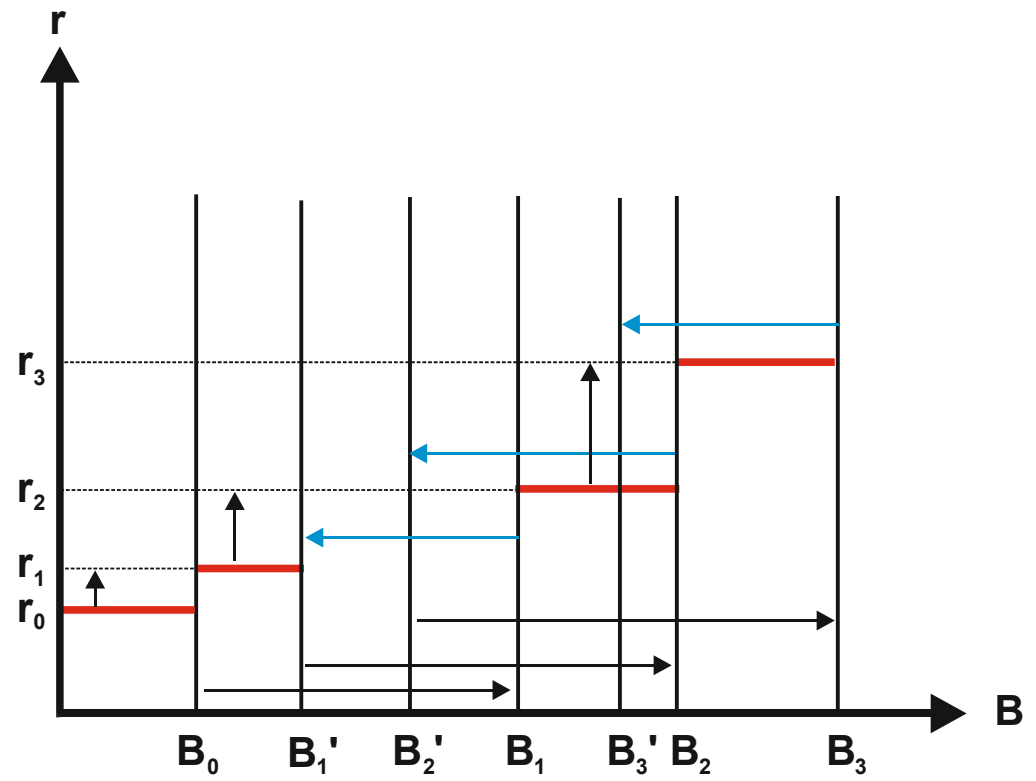

Figure 4. "Return" of the action base through the ASSJ

Source: graphic design of the authors.

Based on the results obtained above, in this paragraph we will propose an automatic stabilizer in the field of social justice (ASSJ). The design of this stabilizer will be based on the following specific design elements:

- the generating activity $(A G)$ of the variation of the action base is the economic activity, by which we understand the lucrative activity (i.e. the profit-producing activity);

- the base of action $(B)$ will be considered the gross income of the factors of production - wage/salary, interest, profit, rent, as the case may be;

- the sensitivity grid $(G)$ will represent a linear grid of institutional type regarding the ratio between the current per capita income of the favoured persons and the current per capita income of the disadvantaged people;

- the rate of action $(r)$ will be represented by the marginal taxation rate of the uninvested wealth to the persons located in the favoured pole of the society. The rationale for choosing such an action rate consists of the following:

$>$ choosing as the rate of action the current income tax rate would have had a possible pro-cyclical effect, as it would have affected both the current income of 
the disadvantaged and the income of the favoured persons; the result could have been, in some cases or even in most cases, a maintenance of economic inequality, measured by the ratio between the current per capita incomes of the two categories of persons in the society;

the choice of wealth as an object of the action rate, although the grid is sensitivity refers to the current income, it is explained as follows: the current income, more precisely the available income, can be used either for current final consumption or for future final consumption, the latter being possible as a result of saving from current income. On the other hand, the savings can be used either for investments (real or portfolio), or for keeping it (treasury) in banks or even in personal stock. But the saving invested has positive effects on the society, including for the disadvantaged people (jobs created either through direct investments or through the financing of entrepreneurs as a result of portfolio investments). On the other hand, uninvested saving does not have such direct positive effects on the economy. However, things are not completely unambiguous in this matter: deposits held in banks or in monetary funds (such as pension funds) can be used by depositors to indirectly finance the national economy, having similar effects to those of saving used directly for investments. Uncertainty about this role (and, especially, the scale at which this role can take place) of uninvested (treasured) savings leads us to consider that this part of the saving should be subject to the rate of action;

$>$ the existence of saving expresses, in itself, the "surplus" enjoyed by the favoured persons in the society, as a result of current income flows, since it is not consumed; at the same time, although the consumption of the favoured persons may be excessive (for example, a very large share of the consumption of luxury goods), we cannot, by virtue of a free society, directly reduce the current income of these persons to decrease this excessive consumption (the Engel curves describe, in a paradigmatic, qualitative way, the consumption behaviour that addresses the three categories of goods - of strict necessity, long-term use and type of services - in terms of their weight in the total final consumption, based on current income), therefore we take into account a "natural” signaller of this surplus, namely the uninvested saving. There are, of course, a number of difficulties, both theoretical and methodological, here: 1) after how much time of non-investment of the saving can be considered that we have to do with an uninvested saving?; 2) does the deposit of the savings to the banks, that is to say its fruition through the interest, plus the possibility that the deposit in question be used for the indirect external financing of the national economy, does not plead for a certain degree of investment of the saving to be considered uninvested? In this study, we will not examine these difficulties.

we do not distinguish between the growth of uninvested savings as a result of the level (or increasing) of current income and its increasing as a result of its own fruition through bank interest or other type of fruition.

Some notations, which will continue to be used:

- $P$ : the total population in a society 
- $F$ : the pole of the favoured persons in the society

- $\bar{F}$ : the pole of unfavoured people in society

- $M$ : the people between the two poles (the so-called middle social class) in society

- $N(F)$ : number of favoured persons

- $N(\bar{F})$ : number of unfavoured people

- $N(M)$ : the number of persons between the two poles

- $N(P)$ : the number of the total population

- $f$ : weight of the number of favoured persons in the total population

- $\bar{f}$ : weight of the number of unfavoured persons in the total population

- $m$ : the weight of the number of persons between the two poles in the total population

- $V$ : the current income of the favoured persons

- $\bar{V}$ : current income of unfavoured people

- $v$ : the current personal income per capita for the favoured persons

- $\bar{v}$ : current personal income per capita for the unfavoured persons

- $k$ : the coefficient of marginal propensity towards saving of the favoured persons

- $\bar{k}$ : coefficient of marginal propensity towards saving of unfavoured persons

- $t$ : the tax rate of the personal current income of the favoured persons

- $\bar{t}$ : the rate of taxation of personal current income of unfavoured persons

- $v_{D}$ : the disposable income per capita of the favoured persons

- $\bar{v}_{D}$ : disposable income per capita of unfavoured persons

- $S$ : saving for favoured persons

- $\bar{S}$ : saving for unfavoured persons

- $s$ : per capita saving for favoured persons

- $\bar{s}$ : per capita savings for unfavoured persons

- $\varepsilon$ : the coefficient of uninvested savings in the case of favoured persons

- $\bar{\varepsilon}$ : the coefficient of uninvested savings in the case of unfavoured persons

- $W$ : uninvested savings in favour of favoured persons

- $\bar{W}$ : uninvested savings in favour of unfavoured persons

- $w$ : saving uninvested per capital for the favoured persons

- $\bar{w}$ : saving uninvested per capital for unfavoured persons

- $\rho$ : marginal non-investment of wealth in the case of favoured persons

- $\bar{\rho}$ : marginal non-investment of wealth in the case of unfavoured persons

- $\varphi$ : marginal investment of the income available to the favoured persons

- $\bar{\varphi}$ : marginal investment of the income available to unfavoured persons

- $\theta$ : the ratio between the gross current income of the favoured persons and the gross current income of the unfavoured persons

- $\lambda$ : the ratio between non-investment in favoured persons and non-investment in the case of unfavoured persons

The calculation relationships are as follows: 
- $N(P)=N(F)+N(\bar{F})+N(M)$

- $f=\frac{N(F)}{N(P)}$

- $\bar{f}=\frac{N(\bar{F})}{N(P)}$

- $m=\frac{N(M)}{N(P)}$

- $f+\bar{f}+m=1$

- $v=\frac{V}{N(F)}$

- $\bar{v}=\frac{\bar{V}}{N(\bar{F})}$

- $v_{D}=(1-t) \cdot v$

- $\bar{v}_{D}=(1-\bar{t}) \cdot \bar{v}$

- $s=k \cdot v_{D}=k \cdot(1-t) \cdot v$

- $\bar{s}=\bar{k} \cdot \bar{v}_{D}=\bar{k} \cdot(1-\bar{t}) \cdot \bar{v}$

- $w=\varepsilon \cdot s=\varepsilon \cdot k \cdot(1-t) \cdot v$

- $\bar{w}=\bar{\varepsilon} \cdot \bar{s}=\bar{\varepsilon} \cdot \bar{k} \cdot(1-\bar{t}) \cdot \bar{v}$

- $\rho=\frac{\Delta w}{\Delta s}$

- $\bar{\rho}=\frac{\Delta \bar{w}}{\Delta \bar{s}}$

- $\varphi=\frac{\Delta w}{\Delta v_{D}}$

- $\bar{\varphi}=\frac{\Delta \bar{w}}{\Delta \bar{v}_{D}}$

The logic scheme based on which the ASSJ proposed by us is designed is shown in figure 5 .

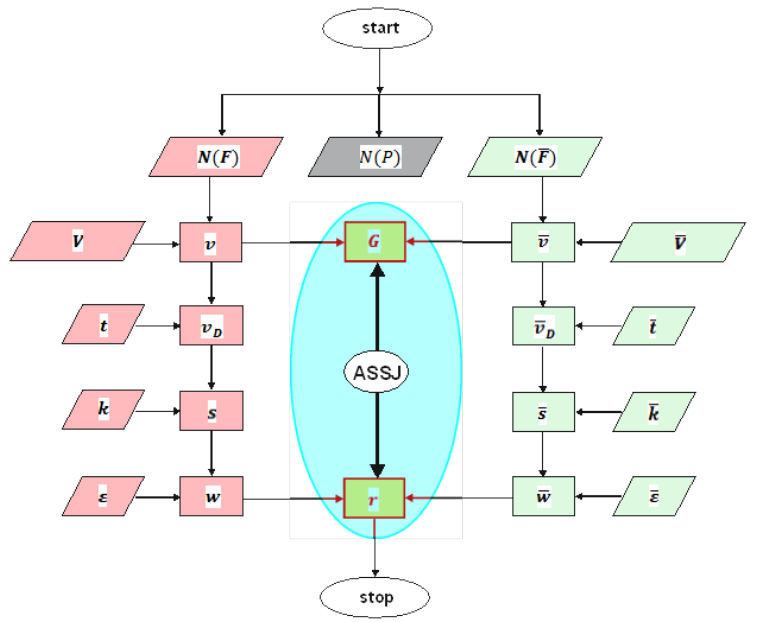

Figure 5. Logical design / redesign scheme of the ASSJ

Source: graphic design of the authors. 
The general mechanism of our proposal for an automatic stabilizer in the field of social justice is synoptically represented in figure 6 .

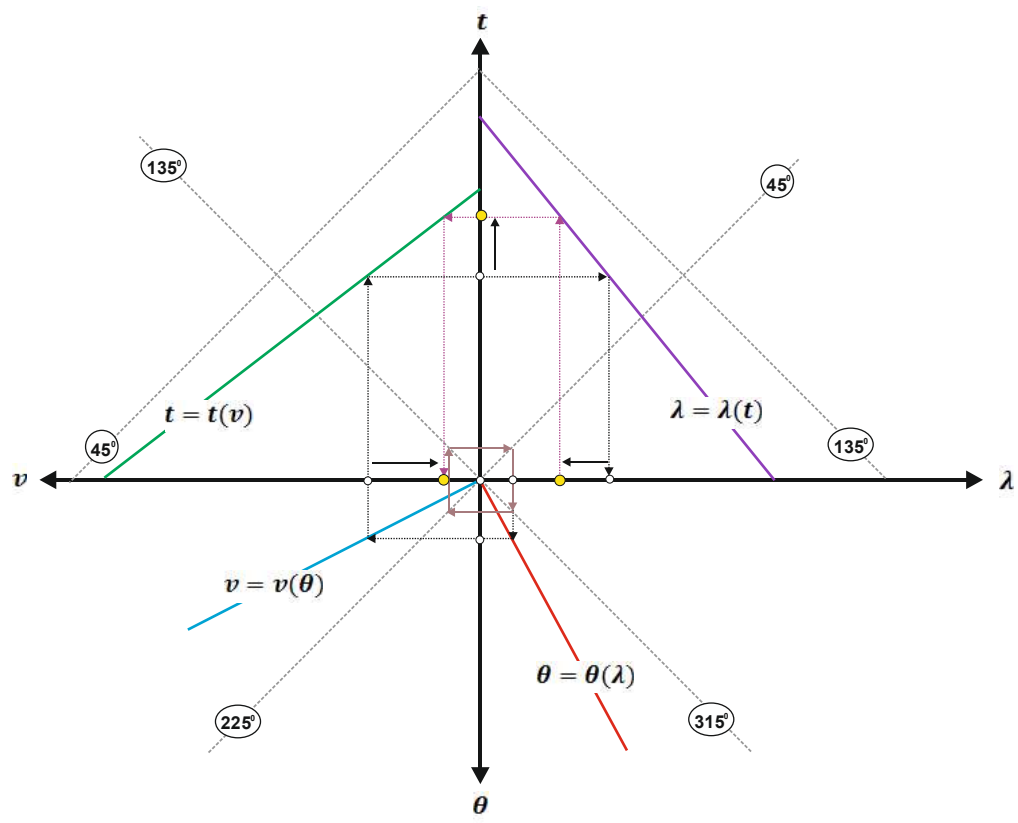

Figure 6. ASSJ mechanism

Source: graphic design of the authors.

\section{References:}

McKay, A., and Reis, R., (2013), The Role of Automatic Stabilizers in the US Business Cycle. Econometrica 84.

Nozick, R., (2007): Anarhie, stat și utopie. Editura Humanitas.

Nussbaum, M., (2007): Frontiers of Justice. Disability, Nationality, Species Membership. The Belknap Press of Harvard University.

Rawls, J., (2011): O teorie a dreptății. Editura Universității „Alexandru loan Cuza”, Iași.

Russell, F., and Kowalewski, K., (2015), „How CBO Estimates Automatic Stabilizers.” Working Paper 2015-07. 